\section{CUSTOMISED IMPLANTOLOGY}

Created with practitioners, the Bien-Air Chiropro L ultra-efficient, extremely versatile implantology system leaves nothing to chance.

The Chiropro L has been designed with efficiency and comfort in mind. The intuitive interface can be managed by the control pedal. The patented peristaltic pump with disposable irrigation lines and insertion support ensures easy handling and optimum infection control.

The Chiropro L System includes the MX-LED self-ventilating motor, which is the most powerful on the market, offering LED lighting at equal intensity at both high and low speeds. Incorporating seven of the leading brands of implants with their complete sequences, the Chiropro L can also be customised to your individual requirements.

With the world's first internal irrigation system the 20:1 contraangle handpiece incorporates the smallest head on the market allowing access to the tightest of areas. Fitted with an exclusive double optical glass rod system, the handpiece provides uniform lighting of the operative field.

This user-friendly system has been proven to meet or surpass the demands of implantologists worldwide.

Reader response number 62

\section{ENTRY LEVEL 3D CONE BEAM}

When practices buy a Galileos Compact from Sident Dental Systems it offers them a convenient entry to the world of 3D cone beam digital diagnostics, with the ability to upgrade for expansion. It also offers unparalleled functionality in diagnosis, planning and treatment because it includes a CEPH facility.

Galileos 3D cone beam digital X-ray systems offer practices the power of integrated diagnostics and treatment planning via a single imaging system.

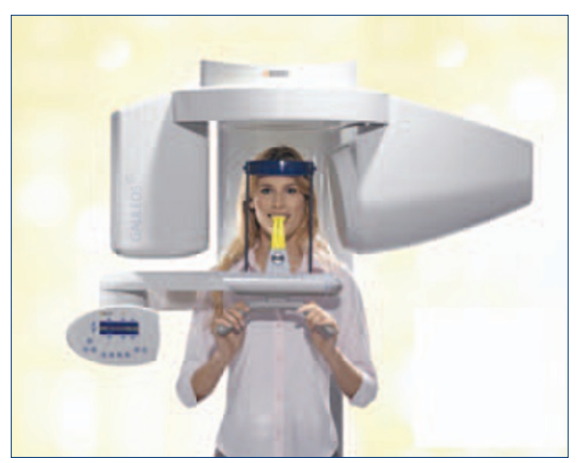

It enables them to reduce risks, plan surgical interventions, coordinate treatment planning with colleagues and explain treatment to patients with even greater clarity, certainty and ease.

With their innovative Cone Beam technology, they can calculate a large volume 3D image set (over 200 exposures) in a single low-dose scan lasting 15 seconds or less. This 3D image set is then processed and displayed using their integrated GALAXIS 3D software.

The Galileos Compact presents its 3D images in perfect image quality and facilitates easy navigation and diagnosis in traditional PAN and TSA presentations. The Galileos Comfort offers CEPH views too. Both are all-in-one diagnosis and planning systems which offer a very short, low dose scanning cycle for optimum speed and efficiency.

Reader response number 63

\section{BETTER RETENTION} OF DAMAGED TEETH

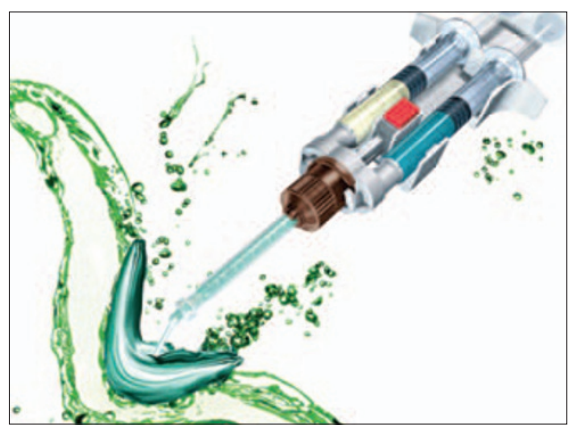

The essential conditions required for implant dentistry can successfully be achieved with Straumann's range of regenerative products.

Straumann Bone Ceramic is a fully synthetic bone substitute with optimised morphology leading the way to new vital bone, offering the clinician flexibility in a broad range of regenerative procedures. Straumann Emdogain is a reliable and clinically proven biology-based treatment solution that helps restore aesthetics and successfully promotes the potential for re-growth of hard and soft tissues lost due to periodontal disease.

Combine the two and you have Straumann Emdogain PLUS which successfully offers the regenerative capabilities of Straumann Emdogain together with the structural stability of Straumann Bone Ceramic. This combination promotes better retention of heavily damaged teeth.

Straumann MembraGel is advanced technology for your guided bone regeneration (GBR) cases. The liquid applicable membrane is designed for precise, simple and quick procedures and when solidified stabilises the bone graft material confining it to the site of the bone augmentation.

Reader response number 64

\section{A FASCINATING EYE-CATCHER}

The CEREC System, from Ceramic Systems, is a computer-aided method that enables dentists to create precision fitting all-ceramic restorations with perfect marginal seals and contours at the chairside. Saving laboratory costs it enables dentists to design and create allceramic inlays, onlays, partial crowns, veneers and crowns for the anterior, premolar and molar regions in one visit. CEREC uses a digital impression taking technique to capture the data required to design the restoration which is then milled from a solid block in the milling unit. The milling unit can be situated anywhere within the practice, even as a fascinating eyecatcher in the waiting room.

In combination with adhesive bonding techniques, CEREC creates biocompatible, non-metallic, naturallooking restorations without the need for provisional restorations. Reader response number 65 